\title{
TENTATIVA DE ERRADICAÇÃO DA HIMENOLEPÍASE EM UMA COMUNIDADE SEMIFECHADA, EMPREGANDO-SE PRAZIQUANTEL
}

\author{
Maria de Lourdes Magalhães de CASTRO (1), José Roberto MACHADo E SILVA (2)
}

e Jorge Luiz Mello SAMPAIO (3)

\begin{abstract}
R E S U M O
Duas semanas após o insucesso da terapêutica com mebendazol - $400 \mathrm{mg}$ diários durante quatro dias consecutivos,- 101 indivíduos de uma comunidade semifechada, 50,5\% infectados por Hymenolepis nana, em sua maioria crianças entre dois e seis anos de idade, foram tratados com praziquantel (*) em duas doses orais de 20 a $25 \mathrm{mg} / \mathrm{kg}$, administradas com dez dias de intervalo. O diagnóstico da himenolepíase, bem como os controles de cura parasitológica realizados nos $7 .^{\circ}, 14 .^{\circ}, 210^{\circ}, 30^{\circ}, 600^{\circ}$ e $90 .^{\circ}$ dias depois da administração da segunda dose de praziquantel, basearam-se em exames de fezes pelo método quantitativo de KATO/KATZ. A tolerância ao medicamento foi excelente e a negativação dos exames ocorreu independentemente da intensidade do parasitismo. Nos 7. e $14 .^{\circ}$ dias pós-tratamento encontraram-se ovos de H. nana, respectivamente em nove e em dois pacientes, mas esses ovos apresentavam-se distorcidos. No controle do 21. dia todos os resultados mostraram-se negativos, traduzindo um índice de cura de $100 \%$. A partir do $300^{\circ}$ dia verificou-se em três crianças a eliminação de ovos normais do parasita. Tendo em vista serem essas as únicas que viviam em regime de semi-internação nessa comunidade e a positividade tardia dos exames, esses casos foram considerados como reinfecção. Conclui-se, pelos resultados alcançados, que o esquema posológico empregado, fundamentado nas investiga. ções experimentais conduzidas por CAMPOS \& col. (1983), é eficaz e seguro para o tratamento da himenolepiase, em especial, quando se pretende tentar erradicá-la numa comunidade fechada.
\end{abstract}

\section{N T R O D U G Ã O}

A himenolepíase é uma parasitose intestinal, causada por qualquer uma das duas espécies de cestóides do gênero Hymenolepis - H. nana, mais encontrada no homem, e H. diminuta ou tênia do rato. Sua distribuição é cosmopolita, sendo observada com maior frequiência nos países de clima quente. Entretanto, inquéritos coproscópicos realizados no Brasil sugerem uma inversão nessa tendência, tendo em vista seu predomínio nos estados sulinos, onde a temperatura média anual é mais baixa.

Ao contrário dos outros cestóides, o H. naua encontra-se com maior frequiência nas crianças, constituíndo-se também no único que necessita de apenas um hospedeiro para seu de. senvolvimento, vez que todo o ciclo biológico do parasita pode completar-se no homem. 0

(*) CESTOX - Merck S. A. Indústrias Químicas

(1) Mestre em Gastrenterologia pela PUC - Rio de Janeiro, RJ

(2) Auxiliar de Ensino da Disciplina de Parasitologia da Fac. de Ciencias Médicas da UERJ, Rio de Janeiro, RJ, Brasil

(3) Monitor da Disciplina de Parasitologia da Fac. de Ciências Médicas da UERJ. Trabalho apresentado no "XVIII Congreso Paranamericano de Gastroenterologia", Guatemala, novembro de 1983. 
CASTRO, M. de L. M. de; MACHADO E SILVA, J. R. \& SAMPAIO, J. L. M. - Tentativa de erradicação da himenolepíase em uma comunidade semifechada, empregando-se praziquantel. Rev. Inst. Med. trop. São Paulo 27:27.32, 1985 .

verme adulto do H. nana mede, em geral, entre 2 e $4 \mathrm{~cm}$ de comprimento e tem seu habitat na porção terminal do íleo. Os ovos são eliminados nas fezes, em condições de infectar a mesma ou outras pessoas. A transmissão processa-se, na maioria das vezes, por via direta, através da ingestão dos ovos embrionados, encontrados nos alimentos e utensilios contaminados ou, ainda, através do contato entre in divíduos parasitados, principalmente crianças vivendo em agrupamentos. Nesses casos o homem funciona como hospedeiro tanto definitivo, da forma adulta, quanto intermediário, da forma larvária ou cisticercóide. Eventualmente a infecção pode ocorrer por via indireta, através da ingestão acidental de pequenos insetos, como pulgas e certos artrópodes coleópteros da farinha, contendo em seu interior formas larvárias do $H$. nana, que se transformam em vermes adultos no intestino do homem. INes. se caso o indivíduo funciona apenas como hospedeiro definitivo. No intestino delgado os embriões, libertados dos ovos ingeridos, penetram nas vilosidades e em cerca de quatro dias desenvolvem-se em larvas cisticercóides. Ao romper a vilosidade, o parasita na forma jovem, fixa-se à mucosa intestinal pelo escólex e, em torno de oito a dez dias atinge a maturidade, iniciando a oviparidade. Alguns casos de parasitismo persistente e intenso podem ser explicados pela auto-infecção externa - ânus, mão, boca - ou interna. Essa última decorre de o homem ser o hospedeiro definitivo e intermediário do H. nana, havendo, portanto, a possibilidade de os ovos no intestino libertarem as larvas que penetrariam nas vilosidades e se desenvolveriam em vermes adultos, sem alcançarem o meio externo ${ }^{1}$. Além da auto-infecção existem outros fatores que dificultam sobre maneira a erradicação da himenolepiase, tais como: precárias eondições de saneamento básico, descuido com a higiene pessoal e, também, a baixa eficácia dos medicamentos disponíveis para seu tratamento.

Até a década de 80 , as poucas drogas destinadas ao tratamento da himenolepíase proporcionavam quase sempre resultados modestos, mesmo quando empregadas em doses elevadas e por tempo prolongado (uma semana).

A niclosamida foi durante muito tempo considerada a medicação de escolha. Entretan. to, além de sua administração exigir medidas especiais (laxantes, dieta etc.), dificilmente ela proporcionava resultados superiores a $80 \% 12$. CAMILLO-COURA \& col. ${ }^{4}$, por exemplo, empregando a niclosamida em dose única, repetida 10 dias após, conseguiram curar somente $50 \%$ dos pacientes, enquanto BARANSKI \& col. ${ }^{2}$, apesar de utilizá-la durante sete dias consecutivos, não alcançaram indices de cura acima de $75 \%$.

O mebenđazole nesta indicação não demonstrou resultados favoráveis. $O$ índice de cura obtido por CASTRO ${ }^{8}$, com dose de $400 \mathrm{mg}$ diários, durante quatro dias, ficou abaixo de $15 \%$ e GOMES ${ }^{9}$, ao empregar posologia seme Ihante, não registrou cura em paciente algum

$O$ advento do praziquantel, derivado sintético pirazino isoquinoleínico, abriu novas perspectivas no tratamento da himenolepíase. Sua eficácia em dose ünica de 20 a $25 \mathrm{mg} / \mathrm{kg}$ de peso foi demonstrada em diversos trabalhos clínicos 7,13,11, conseguindo alguns Autores 14,3 curar todos os indivíduos submetidos ao tratamento.

Entretanto, constatam-se casos que não respondem integralmente a esse medicamento cuja atividade antiparasitária é tanto mais eficaz quanto mais próximo da maturidade encontrase o H. nana. Considerando, por conseguinte, a eficácia relativamente menor do praziquantel sobre as formas larvares, principalmente, os cisticercóides albergados nas vilosidades intestinais, aventou-se a hipótese desse fato ser responsável pelas eventuais falhas terapêticas.

Nesse sentido, CAMPOS \& col. 5 conduziram investigações experimentais visando a estabelecer um esquema posológico capaz de assegurar uma efetividade terapêtica de $100 \%$. Assim, infectaram camundongos com ovos de H. nana, tratando-os com praziquantel na dose de $25 \mathrm{mg} / \mathrm{kg}$, administrada por via oral, em diferentes fases evolutivas do parasita. Verificaram que, ao se empregar a droga no $100^{\circ}$ dia após a infecção, quando o verme já atingiu uma fase em que é inteiramente sensível à ação do praziquantel mas ainda não iniciou a oviparidade torna-se possível erradicar a parasitose. Extrapolando esses resultados, sugeri. ram que o tratamento da himenolepíase humana fosse feito com duas doses num intervalo de 10 dias. Desse modo, a primeira eliminaria 
CASTRO, M. de L. M. de; MACHADO E SILVA, J. R. \& SAMPAIO, J. L. M. - Tentativa de erradicaçäo da himenolepiase em uma comunidade semifechada, empregando-se praziquantel. Rev. Inst. Med. trop. São Paulo 27:27-32, 1985.

os vermes já adultos enquanto a segunda suprimiria aqueles que houvessem escapado ao efeito da primeira por estarem, naquela ocasião, em fases iniciais de seu desenvolvimento.

CAMPOS \& col. ${ }^{6}$ confirmaram na clínica a eficácia desse esquema posológico, ao curar todos os indivíduos portadores de himenolepíase numa determinada comunidade semifechada.

Baseados nos resultados desses estudos, resolvemos utilizar o mesmo esquema posológico, na tentativa de erradicar a himenolepíase numa coletividade onde o índice dessa parasitọse era extremamente elevado e cuja terapia anterior com o mebendazol havia sido desfavorável.

\section{PACIENTES E MÉTODOS}

O estudo foi realizado em um orfanato localizado na zona norte da cidade do Rio de Janeiro, no período de outubro/82 a janeiro/83. A comunidade composta de 101 indivíduos, abrigava 82 crianças entre dois e seis anos e 19 pessoas cuja faixa etária variava de 13 a 48 anos (Tabela I). Essas últimas eram todas do sexo feminino, enquanto $76,8 \%$ das crianças pertenciam ao sexo masculino.

T A B E I A I Distribuição etária

\begin{tabular}{|c|c|c|c|}
\hline \multicolumn{2}{|c|}{ Idade } & \multicolumn{2}{|c|}{ Indivíduos } \\
\hline (em & anos) & N.o & $(\%)$ \\
\hline & 2 & 1 & 1,0 \\
\hline & 3 & 13 & 12,9 \\
\hline & 4 & 23 & 22,8 \\
\hline & 5 & 28 & 27,7 \\
\hline & 6 & 17 & 16,8 \\
\hline & a 15 & 4 & 4,0 \\
\hline & a 19 & 8 & 7,9 \\
\hline & $\equiv 20$ & 7 & 6,9 \\
\hline & otal & 101 & 100,0 \\
\hline
\end{tabular}

Verificou-se, através de exames parasitológicos de fezes efetuados segundo o método de KATO-KATZ ${ }^{10}$, que $50,5 \%(51 / 101)$ da população do orfanato apresentava himenolepíase: 49 crianças e dois adultos (Tabela II). Nenhum outro parasita foi encontrado associado ao $\mathbf{H}$. nana, provavelmente em razão do tratamento da comunidade com o mebendazole, duas semanas antes do início deste estudo. A positi- vidade dos exames foi significativamente maior - teste do qui-quadrado $=\mathrm{p}<0,001$ - entre as crianças, $59,8 \%(49 / 82)$, já que entre os adultos apenas $10,5 \%(2 / 19)$ encontravam-se in. fectados. A carga parasitária verificada antes do tratamento variava entre 24 a 38.456 ovos $/ \mathrm{g}$ de fezes, com uma média de 2.913 e uma mediana de 636. As meninas apresentaram parasitismo mais intenso do que os meninos - teste $\mathrm{t}$ de Student $=\mathrm{p}<0,04$ (Tabela III).

$$
\text { T A B E I A II }
$$

Positividade inicial para H. nana segundo a idade

\begin{tabular}{|c|c|c|c|}
\hline \multirow{2}{*}{$\begin{array}{l}\text { Idade } \\
\text { (em anos) }\end{array}$} & \multirow{2}{*}{$\begin{array}{l}\text { Número } \\
\text { de casos }\end{array}$} & \multicolumn{2}{|c|}{ Casos positivós } \\
\hline & & N.o & $(\%)$ \\
\hline 2 & 1 & 0 & - \\
\hline 3 & 13 & 11 & 84,6 \\
\hline 4 & 23 & 12 & 52,2 \\
\hline 5 & 28 & 18 & 64,3 \\
\hline 6 & 17 & 8 & 47,1 \\
\hline $\bar{z} 6$ & 82 & 49 & $59,8\left(^{*}\right)$ \\
\hline$\equiv 13$ & 19 & 2 & $10,5\left(^{*}\right)$ \\
\hline Total & 101 & 51 & 50,5 \\
\hline
\end{tabular}

T A B E L A III

Carga parasitária inicial segundo o sexo

\begin{tabular}{|c|c|c|c|c|c|c|}
\hline \multirow{2}{*}{$\begin{array}{c}\text { Número de ovos } / \mathrm{g} \\
\text { de fezes }\end{array}$} & \multicolumn{2}{|c|}{ Masculino } & \multicolumn{2}{|c|}{ Feminino } & \multicolumn{2}{|c|}{ Total } \\
\hline & N.o & $(\%)$ & N:o & $(\%)$ & N. ${ }^{\circ}$ & $(\%)$ \\
\hline$<\quad 100$ & 11 & 28,9 & 2 & 15,4 & 13 & 25,5 \\
\hline $100-1000$ & 12 & 31,6 & 4 & 30,8 & 16 & 31,4 \\
\hline $1001-10000$ & 15 & 39,5 & 4 & 30,8 & 19 & 37,2 \\
\hline$>10000$ & 0 & - & 3 & 23,0 & 3 & 5,9 \\
\hline Total & 38 & 100,0 & 13 & 100,0 & 51 & $100 ; 0$ \\
\hline Média & \multicolumn{2}{|c|}{$1680\left(^{*}\right)$} & \multicolumn{2}{|c|}{$6520\left(^{*}\right)$} & \multicolumn{2}{|c|}{2913} \\
\hline Mediana & \multicolumn{2}{|c|}{588} & \multicolumn{2}{|c|}{3216} & \multicolumn{2}{|c|}{636} \\
\hline Limites & \multicolumn{2}{|c|}{24 a 8076} & \multicolumn{2}{|c|}{24 a 38456} & \multicolumn{2}{|c|}{24 a 38456} \\
\hline
\end{tabular}

(*) $\mathrm{p}<0,04$

Após determinar-se o número de pessoas infectadas todos os membros da comunidade, infectados ou não, foram tratados com o praziquantel na dose oral de 20 a $25 \mathrm{mg} / \mathrm{kg}$ de peso corporal, administrada em uma única tomada e repetida após 10 dias de intervalo. Durante $o$ tratamento nenhuma medida especial (dieta, jejum etc.) se fez necessária, tampouco empregou-se qualquer outro tipo de medicamento (laxantes, antieméticos etc.).

Para o controle de cura parasitológica dos indivíduos infectados e acompanhamento dos 
CASTRO, M. de L. M. de; MACHADO E SILVA, J. R. \& SAMPAIO, J. I. M. - Tenitativa de errađicaçăo da himenolepíase em uma comunidade semifechada, empregando-se praziquantel. Rev. Inst. Med. trop. São Paulo 27:27-32, 1985.

não infectados, foram realizados exames coproscópicos nos $7 .^{\circ}, 14 .^{\circ}, 21 .^{\circ}, 30 .^{\circ}, 60^{\circ}$ e $90 .^{\circ}$ dias após a segunda dose do praziquantel, adotando-se o mesmo método utilizado para o diag nóstico da himenolepíase.

$O$ resultado de cada exame representou a média aritmética do número de ovos encontrados em três alíquotas de fezes, colhidas em cada controle.

Visando a acompanhar a evolução dos pacientes, inclusive detectar possiveis efeitos adversos da medicação, todos os indivíduos da comunidade foram submetidos a exames médicos periódicos, coincidentes com a época do tratamento e dos controles parasitológicos.

\section{RESULTADOS}

Dos 51 pacientes infectados unicamente nove crianças, quatro meninas e cinco meninos, com idades variando entre quatro e seis anos, apresentaram positividade no $10^{\circ}$ controle $\left(7 .^{\circ}\right.$ dia) após o tratamento e, dessas, só duas permaneceram positivas no $2 .^{\circ}$ controle $\left(14 .^{\circ} \mathrm{dia}\right)$. No $3 .^{n}$ controle (21. dia), entretanto, todos haviam negativado seus exames. A Tabela IV apre. senta os resultados desses controles parasitológico pós-tratamento dos indivíduos infectados; já que os não infectados, compreendendo $49,5 \%$ (50/101) da população, mantiveram-se negati vos até o último controle (90. dia).

T A B E L A IV

Resultados dos exames de fezes após o tratamento

\begin{tabular}{|c|c|c|c|c|}
\hline \multirow{2}{*}{$\begin{array}{c}\text { Epoca do controle } \\
\text { pós-tratamento }\end{array}$} & \multicolumn{2}{|c|}{ Casos negativos } & \multicolumn{2}{|c|}{ Casos positivos } \\
\hline & N.o & $(\%)$ & N.O & $(\%)$ \\
\hline 7. ${ }^{\circ}$ dia & 42 & 82,4 & 9 & 17,6 \\
\hline 14. dia & 49 & 96,1 & 2 & 3,9 \\
\hline $21 .^{c}$ dia & 51 & 100,0 & 0 & - \\
\hline $30 .^{\circ} \mathrm{dia}$ & 47 & 92,2 & 4 & $\mathbf{7 , 8}$ \\
\hline $60.0^{\circ} \mathrm{dia}$ & 48 & 94,1 & 3 & 5,9 \\
\hline $900^{\circ}$ dia & 48 & 94,1 & 3 & 5,9 \\
\hline
\end{tabular}

Durante a realização das coproscopias verificou-se nítida alteração entre a morfologia dos ovos antes e após o tratamento. A Tabela $\mathrm{V}$ mostra o resultado desses exames, seguncio o aspecto morfológico dos ovos de $\boldsymbol{H}$. nana. Observou-se que nos dois primeiros controles após o tratamento $\left(7 .^{\circ}\right.$ e $\left.14 .^{\circ} \mathrm{dia}\right)$ todos os ovos encontrados apresentavam-se distorcidos. A partir do $300^{\circ}$ dia, no entanto, excetuando-se uma criança que ainda eliminou ovos distorcidos, nas outras três que voltaram a ter exames positivos somente foram detectados ovos normais.

T A B E L A V

Carga parasitária e aspecto morfológico dos ovos de H. nana nas nove crianças com exames positivos após o tratamento

\begin{tabular}{|c|c|c|c|c|c|c|c|c|}
\hline \multirow{2}{*}{ Antes } & \multirow{2}{*}{ do } & \multirow{2}{*}{ tratamento } & \multicolumn{6}{|c|}{ Controles pós-tratamento } \\
\hline & & & 7.० dia & $14 .^{\circ}$ dia & $21 .{ }^{\circ} \mathrm{dia}$ & $300^{\circ}$ dia & $60.0^{\circ}$ dia & $90^{\circ}$ dia \\
\hline & $\mathbf{N}$ & $(38455)$ & $\mathrm{D}(24)$ & $\mathrm{D}(24)$ & - & $N(24)$ & $N(24)$ & N (24) \\
\hline & $\mathbf{N}$ & $(9910)$ & $\mathrm{D}(24)$ & - & - & $\mathrm{N}(24)$ & $N(24)$ & $N(24)$ \\
\hline & $\mathbf{N}$ & $(48)$ & $D(72)$ & 一 & - & $N(24)$ & $N(24)$ & $N(24)$ \\
\hline & $\mathrm{N}$ & $(10912)$ & $\mathrm{D}(24)$ & $\mathrm{D}(24)$ & - & $\mathrm{D}(24)$ & - & - \\
\hline & & ( 144) & $\mathrm{D}(24)$ & - & - & - & - & - \\
\hline & & ( 2928$)$ & D ( 24) & - & - & - & - & - \\
\hline & $\mathrm{N}$ & ( 5368$)$ & $\mathrm{D}(24)$ & 一 & - & - & - & - \\
\hline & $\mathrm{N}$ & ( 5784$)$ & $D(72)$ & - & - & - & - & - \\
\hline & $\mathrm{N}$ & $(2878)$ & $D(120)$ & - & - & 一 & - & - \\
\hline Média & & $(8492)$ & ( 45) & (24) & $(0)$ & $(24)$ & $(24)$ & (32) \\
\hline
\end{tabular}

$\mathrm{N}=$ ovos normais; $\mathrm{D}=$ ovos distorcidos

0 ) número de ovos/g de fezes

A negativação dos exames coproscópicos ocorreu independentemente da intensidade do parasitismo, embora a carga parasitária média (8.492) das nove crianças positivas pós-trata- mento fosse, antes do mesmo, significativamen. te maior - teste U de MANN-WHITNEY = $\mathrm{p}<0.01$ - do que a das outras crianças $(1.718$ ovos). 
CASTRO, M. de L. M. de; MACHADO E SILVA, J. R. \& SAMPAIO, J. L. M. - Tentativa de erradicação da himenolepiase em uma comunidade semifechada, empregando-se praziquantel. Rev. Inst. Med. trop. São Paulo 27:27-32, 1985.

A Tabela VI apresenta resultado final do tratamento, levando em consideração não só a época da positividade dos exames, mas também a viabilidade dos ovos.

T A B E L A VI

Avaliação parasitologica final

\begin{tabular}{lcr}
\hline Pacientes & N. ${ }^{\circ}$ & $(\%)$ \\
\hline Curados & 51 & $\mathbf{1 0 0 , 0}$ \\
Reinfectados & 3 & $\mathbf{5 , 9}$ \\
\hline
\end{tabular}

A tolerância ao praziquantel foi excelente não sendo relatado qualquer tipo de efeito colateral relacionado ao tratamento.

\section{DISGUSSĀO}

Conforme mencionamos anteriormente, este trabalho foi motivado pela necessidade de erradicarmos a himenolepíase de uma comunidade semifechada, com taxa excessivamente elevada dessa parasitose: 50,5\% (51/101). Além disso, despertou-nos enorme interesse a possibilidade de reproduzirmos neste estudo os resultados obtidos por CAMPOS \& col. 6 , ao empregarem o praziquantel na dose oral de 25 $\mathrm{mg} / \mathrm{kg}$, administrada em uma só tomada e repetida após 10 dias de intervalo, numa comunidade semelhante.

Embora tenhamos alcançado resultados excelentes, curando todos os indivíduos, faz-se necessário analisar com maior profundidade os parâmetros utilizados nos critérios de cura da himenolepiase, no sentido de, também, se identificar as características dos ovos encontrados nos exames de fezes.

Durante a realização dos exames cóproscópicos constatamos que os ovos de H. nana nas nove crianças positivas no $7 .^{\circ} \mathrm{dia}$, bem como nas duas que permaneceram eliminando ovos no $14 .^{\circ}$ dia após o tratamento, apresentavam-se distorcidos. Essas alterações, referidas igualmente por PAZ \& col. 12 e BARANSKI \& col. ${ }^{3}$, provavelmente decorrem da ação do praziquantel sobre os órgãos reprodutivos dos parasitas que, no momento do tratamento, encontravamse em processo final de maturação antes de iniciarem a oviparidade, e não de uma atuação direta da droga sobre o ovo já constituído.

O fato de, somente a partir do $30^{\circ}$ dia depois do tratamento, se ter verificado a presen- ça de ovos normais nos exames de três criancas, justamente as únicas da comunidade vivendo em regime de semi-internação, levou-nos a admitir que as mesmas tivessem se reinfectado. A outra criança positiva no $30 .^{\circ}$ dia e negativa nos controles subsequientes foi considerada curada, por ter eliminado exclusivamente ovos distorcidos. Esses foram interpretados como oriundos de parasitas jovens, atingidos pelo medicamento numa fase em que estavam iniciando o processo de formação e postura de ovos. Para investigar se tais alterações morfológicas, observadas nos ovos após o tratamento, seriam indiretamente induzidas pela ação da droga sobre o aparelho reprodutor do parasita em fase final de maturação, consideramos conveniente elaborar um novo ensaio clínico. Esse abrangeria individuos de uma comunidade fechada, altamente parasitados, consequentemente, com probabilidade de albergarem o H. nana em diferentes estágios evolutivos. Efetuando-se exames parasitológicos diariamente até, no mínimo, 21 dias após a administração da segunda dose do praziquantel, poder-se-ía, então, acompanhar o comportamento dos achados coproscópicos.

A cura em $100 \%$ dos pacientes permite-nos concluir que o esquema terapèutico utilizando o praziquantel em dose oral de 20 a $25 \mathrm{mg} / \mathrm{kg}$, repetida 10 dias depois, consiste num modo seguro e eficaz de combate à himenolepíase, sendo também o meio mais adequado para erradicá-la de uma comunidade fechada.

\section{SUMMARY}

Attempt at erradicating hymenolepiasis within a close community using praziquantel

Two weeks after the unsuccessful treatment with mebendazole - $400 \mathrm{mg}$ daily during four consecutive days -, 101 persons living at a semi-close community, $50.5 \%$ infected with Hymenolepis nana, mostly children from 2 to 6 years old, were treated with praziquantel, two single oral doses of 20 to $25 \mathrm{mg} / \mathrm{kg}$ administered ten days apart.

The diagnosis of hymenolepiasis as well as the parasitological controls of cure performed on the 7 th, 14th, 21st, 30th, 60th and 90th days after the second dose administration, were ba- 
CASTRO, M. de L. M. de; MACHADO E SILVA, J. R. \& SAMPAYO, J. L. M. - Tentativa de erradicação da himenolepíase em uma comunidade semifechada, empregando-se praziquantel. Rev. Inst. Med. trop. São Paulo 27:27-32, 1985.

sed on stool examinations according to the quantitative Kato/Katz method.

The drug tolerance was excellent and the negativation of the stool examinations occurred independently from the initial worm burden. H. nana eggs were found on the 7 th and 14 th days post-treatment in nine and in two patients, respectively. However, these eggs were distorted. In the control made on the 21st day all results were negative indicating a $100 \%$ cure rate.

From the 30th day on normal eggs were detected in three children. Considering the late positivity of their examinations and that they were the only persons living part-time outside the community, such cases were classified as reinfection.

By virtue of these results it was concluded that this dose schedule, based on the experimental investigations undertook by CAMPOS et al. (1983), is safe and 100\% efficacious for treating hymenolepiasis, particularly envisageing its erradication in close communities.

\section{AGRADECIMENTOS}

Ao Dr. Getúlio L. de Rezende, Depto. de Pesquisa Clínico-Farmacológica da MERCK S.A. Indústrias Químicas, pelas sugestōes relativas à Metodologia da Pesquisa.

\section{REFERENGIAS BIBLIOGRAFIGAS}

1. BARANSKI, M, C. - Helmintiases intestinais, In: NEVES, J. - Doenças Infectuosas e Parasitárias em Pediatria. Rio, Guanabara. Koogan, 1981, 613-615.

2. BARANSKI, M. C. \& GODOY, O. F. - Tratamento da himenolepíase nana com um derivado da salicilamida(n-(2'-cloro-4'-nitrofenil)-5-clorosalicilamida). Hospital (Rio) 67: 593-599, 1965.

3. BARANSKI, M. C.; GOMES, N. R.; GODOY, O. F.; SILVA, A. F.; KOTAKA, P. I.; GIOVANNONI, M. \& CARNEIRO FILHO, M. - Terapêtutica da teníase e da himenolepíase nana com dose oral única de praziquantel. Estudo da eficácia, tolerância e segurança. Rev. Inst. Medi, trop. São Paulo 22: 82-88, 1980.

4. CAMILLO-COURA, L.; SOLI, A. V.; CARVALHO, H. T. \& SILVA, J. R. - Tratamento da teniase por Hyme. nolepis nana com um derivado da salicilamida (n-(2' cloro-4'-nitrofenil)-5-clorosalicilamida). Hospital (Rio) 69: $93-98,1966$.

5. CAMPOS, R.; VIEIRA BRESSAN, M. C. \& EVANGELISTA, M. G. B. F. - Estudos sobre a ação do praziquantel em camundongos experimentalmente infestados com Hymenolepis nana. VI Congreso de la Federación Latinoamericana de Parasitólogos. São Paulo, setembro, 1983.

6. CAMPOS, R.; MOREIRA, A. A. B.; PINTO, P. L.; AMATO NETO, V.; LEITE, R. M. \& SANT'ANA, E. J. - Tentativa de controle de himenolepíase em coletividade semifechada. VI Congreso de la Federación Latinoamericana de Parasitólogos. São Paulo, setembro, 1983.

7. CaRvalho, S. A.; CAMPOS, R.; amato NeTO, V. \& CASTILHO, V. L. P. - Tratamento, por meio do praziquantel, da infecção humana devida à Hymenolepis nana. Rev. Inst. Med, trop. São Paulo 23: 79$81,1981$.

8. CASTRO, M. L. M. - Enteroparasitoses em um orfanato da cidade do Rio de Janeiro: Aspectos da prevalência, transmissão, quadro clínico e terapêutica. Pontifícia Universidade Católica do Rio de Janeiro. [Dissertação de Mestrado, 1982].

9. GOMES, M. C, O. - Tratamento da teníase pelo mebendazole. F. Méd. (Br.) 66: 1053-1051, 1973.

10. KATZ, N.; CHAVES, A. \& PELLEGRINO, J. - A simple device for quantitative stool thick-smear technique in Schistosomiasis mansoni. Rev. Inst. Med. trop. São Paulo 14: $397-400,1972$.

11. LOUZADA, G. L.; LOUZADA, F. Z.; LOUZADA, T. Z \& BERLITZ, M. F. - Tratamento da teniase e himenolepiase com dose única por via oral de praziquantel. F. Méd. (Br.) 79: 323-326, 1979.

12. PAZ JÁCOME, G.; MENA, H. O. \& DOMMERQUE, F, S. - Efecto terapéutico del prazicuantel en la himenolepiasis. Trib. Méd. 54: 18-20, 1981.

13. PEDRO, R. J.; DEBERALDINI, E. R.; DIAS, L. C. S. \& GOTO, M. M. F. - Tratamento de escolares com Hymenolepis nana pelo praziquantel. Rev. Ass. Med. Brasil. 28: 216-217, 1982.

14. SCHENONE, H.; GALDAMES, M.; RAVADENEIRA, A.; MORALES, E.; HOFFMANN, M. T.; ASALGADO, N.; MENESES, G.; MORA, M. V. \& CABRERA, G. - Tratamiento de las infecciones por Hymenolepis nana en niños con una dosis oral única de prazicuantel (Embay 8440). Bol. Chile. Parasit. 32: 11-13, 1977.

Recebido para publicação em $7 / 2 / 1984$.

Endereço do Autor: Rua Teodoro da Silva, 712 a. 802 20.560 - Rio de Janeiro, RJ, Brasil 